\title{
Bone marrow mesenchymal stem cells combined with Sox 2 increase the functional recovery in rat with traumatic brain injury
}

Qiang $\mathrm{HaO}^{1}$, Jian Zheng ${ }^{2}$, Yue Hu$^{3}$ and Hao Wang ${ }^{1 *}$

\begin{abstract}
Background: About 10 million individuals suffer from traumatic brain injury (TBI) each year in the world, which is one of the most serious neurological disorders. The morbidity of TBI is 55.5 6.1/100,000 in China, which takes more costing in the therapy, and the outcome of that is not well. Therefore, we expect to find new methods to treat TBI and improve the outcomes of TBI. In the previous studies reviewed, we found that stem cell transplantation may hold promising potential for modifying motor dysfunction induced by TBI.
\end{abstract}

Methods: Twenty-six adult SD rats were involved in our study. Two adult SD rats were used as donors of bone marrow stem cells (BMSCs), and the other adult SD rats were divided into four groups randomly, which were used to establish the TBI models. BMSCs were transduced with lentivirous-Sox2, and we try to examine the effects of Sox2 on the differentiation of BMSCS.

Establishment of rat TBI model: Rats were anesthetized using pentobarbital sodium (at a concentration of 1.5\% and a dose of $40 \mathrm{mg} / \mathrm{kg}$ ) and fixed under the stereotaxic device. A 1.0-cm craniotomy was performed lateral to the sagittal suture. The skullcap was carefully removed, and rats were then subjected to TBI using a controlled cortical injury instrument. A standardized parietal contusion was performed using a 20-mg steel rod with a diameter of 4 $\mathrm{mm}$, which dropped from a height of $30 \mathrm{~cm}$. After injury, the incision was sutured, and rats were carefully observed and nursed.

Treatments: Seven days after TBI, rats were divided into four groups and were transplanted with BMSC-Sox2, single BMSC, single Lentivirus-Sox2, and PBS into injured brain, respectively. The motor function was tested using the neurological severity score (NSS).

Results: We found that the ectopic expression of Sox2 enhanced BMSCs to differentiate into neurons. Seven days after TBI, the rats were treated with BMSC-Sox2, BMSC, Sox2, and PBS. Results showed that NSS were $3.352 \pm 0.398$ in the BMSC-Sox2 group, $4.013 \pm 0.495$ in the BMSC group, $4.968 \pm 0.293$ in the Sox 2 group, and $6.257 \pm 0.361$ in the PBS group, suggesting that there were obvious improvements in the neurological function in BMSC-Sox2, BMSC, and Sox2 groups. In addition, the BMSC-Sox2 group had the lowest scores, $p<0.05$.

Conclusion: The ectopic expression of Sox2 could enhance BMSCs to differentiate into neurons, and intervention of BMSCs combined with Sox2 transplantation could promote recovery of motor function in rats with TBI.

Keywords: Traumatic brain injury, Bone marrow stem cell, Sox2 gene, Transplantation

\footnotetext{
* Correspondence: cmu990103@163.com

'Department of Neurosurgery, Beijing Tiantan Hospital, Capital Medical

University, No.119 South 4th Ring West Road, Fengtai District, Beijing 100070,

China

Full list of author information is available at the end of the article
}

(c) The Author(s). 2019 Open Access This article is distributed under the terms of the Creative Commons Attribution 4.0 International License (http://creativecommons.org/licenses/by/4.0/), which permits unrestricted use, distribution, and reproduction in any medium, provided you give appropriate credit to the original author(s) and the source, provide a link to the Creative Commons license, and indicate if changes were made. The Creative Commons Public Domain Dedication waiver (http://creativecommons.org/publicdomain/zero/1.0/) applies to the data made available in this article, unless otherwise stated. 


\section{Background}

About 10 million individuals suffer from traumatic brain injury (TBI) each year in the world, which is one of the most serious neurological disorders. And approximately 70,000 90,000 patients underwent long-term neurological disabilities, which will lead to great medical cost and spending more time to conduct rehabilitative care. In addition, Professor Jiang reports the morbidity of TBI is 55.5-6.1/100,000 in China, which takes more costing in the therapy, and the outcome is not well [1-4]. Unfortunately, current options for treating brain injury are limited. Therefore, to explore promising methods for treating TBI is essential and critical.

However, cell transplantation brings hope to treat TBI. As reliable cell resources, bone marrow stem cells (BMSCs) may provide great promise for regenerative medicine. Firstly, BMSCs have the ability of proliferation in vitro; secondly, they own the ability of differentiation into osteocytes, cartilages, and adipocytes [5]. In addition, it has been reported that BMSCs also have the ability of transdifferentiation into neurons and astrocytes in some reports. However, the ability of BMSCs to transdifferentiate into neurons is limited [6].

Sox2 is a transcription factor, which is highly essential in keeping the ability of self-renewal and pluripotency in embryonic stem cells (ECs) according to previous reports [7]. Sox2-positive neural stem cells (NSCs) not only proliferate as NSCs, but also differentiate into neural precursors $[8]$.

Therefore, we study the effects of ectopic expression of Sox 2 on BMSCs and explore the possibility of BMSC differentiation into neurons. In our study, we try to explore the possibility that BMSC transplantation may lead to therapeutic effects in the improvement of motor dysfunction. The goal of this study is to explore the effects of BMSC transplantation with Sox2 in a rat TBI model.

\section{Methods}

Experimental animals

Adult SD rats (210-250 g, 10-12 weeks old) were employed in accordance with the Capital Medical University (CMU) guidelines and were fed in the animal room under a 12-h light and dark cycle. The minimum number of rats was required according to statistical analysis. Twenty-six adult SD rats were involved in our study. Two adult SD rats were used as donors of BMSCs. The other adult SD rats were divided into four groups randomly and were used to establish the TBI models.

\section{Preparation BMSCs}

BMSCs were extracted from femurs and cultured in basic culture medium (MEM, Gibco, USA), which was added into $1 \mathrm{mM}$ glucose, $17 \%$ fetal bovine serum (FBS, Gibco, USA), $100 \mathrm{U} / \mathrm{ml}$ penicillin, $100 \mu \mathrm{g} / \mathrm{ml}$ streptomycin, and $2 \mathrm{mM}$ glutamine. When cells were cultured for $24 \mathrm{~h}$, the non-adherent cells were removed through changing fresh culture medium. The remaining cells were passaged and stored for future use (Fig. 1).

\section{Flow cytometer}

BMSCs were stained with antibodies to CD34 (Abcam, Ab81289, USA) when passaged to the sixth generation and characterized by FACS analyses. After the cells reached $85 \%$ confluence, they were digested using $0.25 \%$ trypsin-EDTA and analyzed using a flow cytometer to detect the rate of CD34-positive cells.

\section{Differentiation of BMSCs into adipogenic and osteogenic cells}

For the induction of BMSCs into adipogenic and osteogenic cells, the classical protocol was applied as previously described [5]. Briefly, $5 \times 10^{5}$ BMSCs were seeded per well and cultured in an incubator under $37^{\circ} \mathrm{C}$ with
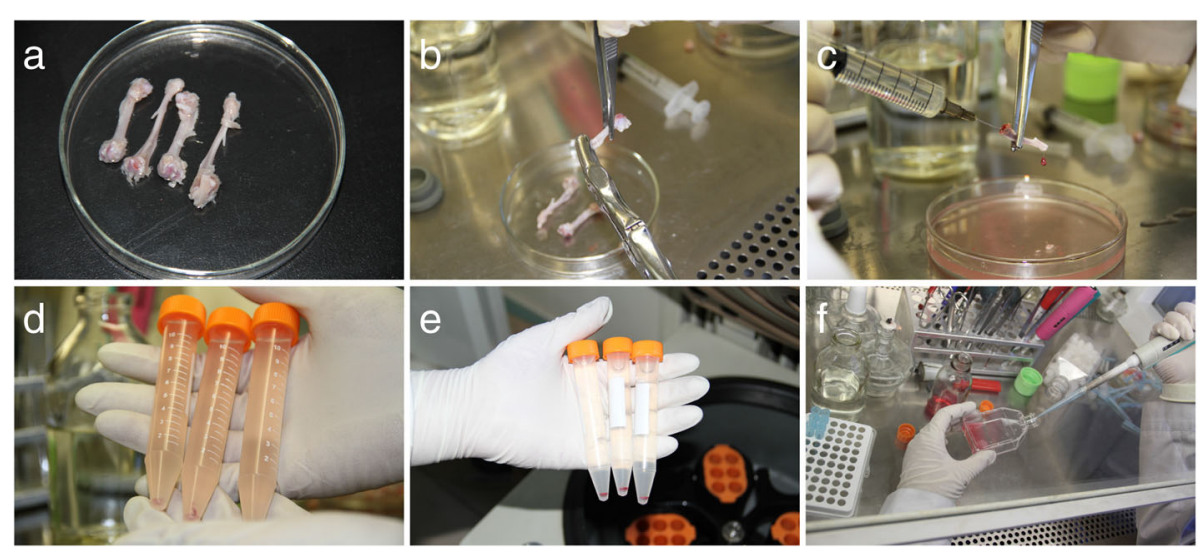

Fig. 1 Procedure of extraction of bone marrow stem cells (BMSCs) from rat. a Obtaining humerus from rats. $\mathbf{b}, \mathbf{c}$ Extraction of bone marrow from the humerus. $\mathbf{d}$, e Bone marrow was made into a suspension and centrifuged. $\mathbf{f}$ Prepare a suspension and inoculate it in a Petri dish 
5\% $\mathrm{CO}_{2}$. After the BMSCs reached $100 \%$ confluence, we changed the culture medium into osteogenetic and adipogenetic differentiation medium, and cultured for additional 3 weeks. Osteogenetic differentiation medium: $\alpha$-MEM, 15\% FBS, $0.1 \mathrm{mM}$ glumax, $0.1 \mathrm{mM}$ antimycotic, $50 \mu \mathrm{g} / \mathrm{ml} \mathrm{L}$-ascorbic acid 2-phosphate, $1 \mathrm{nM}$ dexamethasone, and $20 \mathrm{mM} \beta$-glycerolphosphate. Adipogenetic differentiation medium: $\alpha$-MEM, $15 \%$ FBS, $0.1 \mathrm{mM}$ glumax, $50 \mu \mathrm{M}$ indomethacin, $0.1 \mathrm{mM}$ antimycotic, $0.5 \mu \mathrm{M}$ dexamethasone, and $0.5 \mu \mathrm{M}$ isobutylmethylxanthine. Three weeks after induction, BMSCs were rinsed by PBS and then fixed by formalin for $1 \mathrm{~h}$ at RT. BMSCs for osteogenic differentiation were stained by Alizarin Red S for 20 min at RT, and cells of adipogenic differentiation were stained with oil red $\mathrm{O}$ for $20 \mathrm{~min}$. The cells were observed using a Zeiss inverted microscope.

\section{Lentivirus transduction}

For lentivirus infection, BMSCs were seeded at a concentration of $4 \times 10^{4}$ cells per culture well and then were infected with Sox2-hrGFP-DU3 lentiviruses, supplemented with $0.1 \mathrm{~g} / \mathrm{ml}$ Polybrene (Sigma-Aldrich) for 24 $h$. Lentivirus mediums were washed away using the new culture medium.

\section{Differentiation of BMSCs into neuron-like cells}

The BMSCs were cultured in a culture medium (containing 15\% FBS, $0.1 \mathrm{mM}$ penicillin, $0.1 \mathrm{mM}$ streptomycin, and $0.1 \mathrm{mM}$ glumax) for 4 days, and then the medium was changed into neural induction culture medium, which included the neural induction medium: $50 \mathrm{ng} / \mathrm{ml} \mathrm{B27}$, basic FGF $50 \mathrm{ng} / \mathrm{ml}$, FGF8 $100 \mathrm{ng} / \mathrm{ml}$, and SHH $250 \mathrm{ng} / \mathrm{ml}$. Then, the medium was changed into neural induction medium and BMSCs were cultured for another 10 days. The neuronal induction medium was changed every 2 days.

\section{Immunofluorescence staining}

BMSCs were cultured at a density of 500 cells per culture well and cultured on chamber slides under culture medium. Seven days later, BMSCs were fixed using a formaldehyde solution (Sigma-Aldrich, USA) at RT for 20 min. The cells were blocked with 15\% normal goat serum (Vector, Burlingame, CA, USA) in PBS and coated with primary antibody anti-Tuj1 (Millipore, MA, USA) at $4{ }^{\circ} \mathrm{C}$ for $12 \mathrm{~h}$. Then, the cells were incubated with goat anti-Rabbit lgG (Vector, Burlingame, CA, USA) at 1:200 dilutions for $1 \mathrm{~h}$ at RT. Nuclei were counterstained with DAPI (DAKO, Japan). The BMSCs were observed using a Zeiss inverted fluorescent microscope (Carl Zeiss, Germany).

\section{Weight-drop TBI model}

Establishment of rat TBI model was performed as described by Kalish et al. with some alterations [9]. Briefly, rats were anesthetized using pentobarbital sodium (at a concentration of $1.5 \%$ and a dose of $40 \mathrm{mg} / \mathrm{kg}$ ) and fixed under the stereotaxic device. A 1.0-cm craniotomy was performed lateral to the sagittal suture. The skullcap was carefully removed, and rats were then subjected to TBI using a controlled cortical injury instrument. A standardized parietal contusion was performed using a 20-mg steel rod with a diameter of $4 \mathrm{~mm}$, which dropped from a height of $30 \mathrm{~cm}$. After injury, the incision was sutured, and rats were carefully observed and nursed.

\section{Transplantation}

The TBI rats were divided into four groups: BMSC transplantation group $(n=8)$, Sox2 transplantation group $(n=8)$, BMSCs-Sox 2 transplantation group $(n=$ $8)$, and sham operation group $(n=8)$. BMSCs were prepared at a density of $1 \times 10^{5}$ cells $/ \mu$ l in culture medium and stored on an ice plate. When TBI rats were established for 7 days, $50 \mu \mathrm{l}$ of BMSCs, Sox2, and BMSCs-Sox2 suspension was injected into the injured brain area using a 50- $\mu$ l Hamilton syringe. The same volume of PBS was given to the sham operation group in the same way.

\section{Neurological function testing}

The rats were tested for motor function referring to the neurological severity score (NSS), which is described by Zhao et al., and were graded for the aspect of neurological function [10]. The relative methods are described in Fig. 6. All the rats underwent behavioral tests before TBI, 7 days after TBI, and 3 and 7 days after treatment by BMSCs, Sox2, and BMSCs-Sox2. The more severe the neurological deficit, the scores were higher. The whole procedure is described in Fig. 2.

\section{Statistical analysis}

Statistical results of the data were analyzed by SPSS 16.0 software (USA). Data were expressed as mean \pm SD. When the $p$ value was less than 0.05 , it was considered that there were significant differences between the different groups.

\section{Results}

\section{Characteristics of BMSCs}

BMSCs were selected by FACS and displayed spindle-like in the growth medium. We clarified that BMSCs had the ability of differentiation into adipocytes and osteoblasts. Results showed that Alizarin red S- and Oil red O-positive cells appeared after 3 weeks in the respective culture medium, and BMSCs cultured in the 


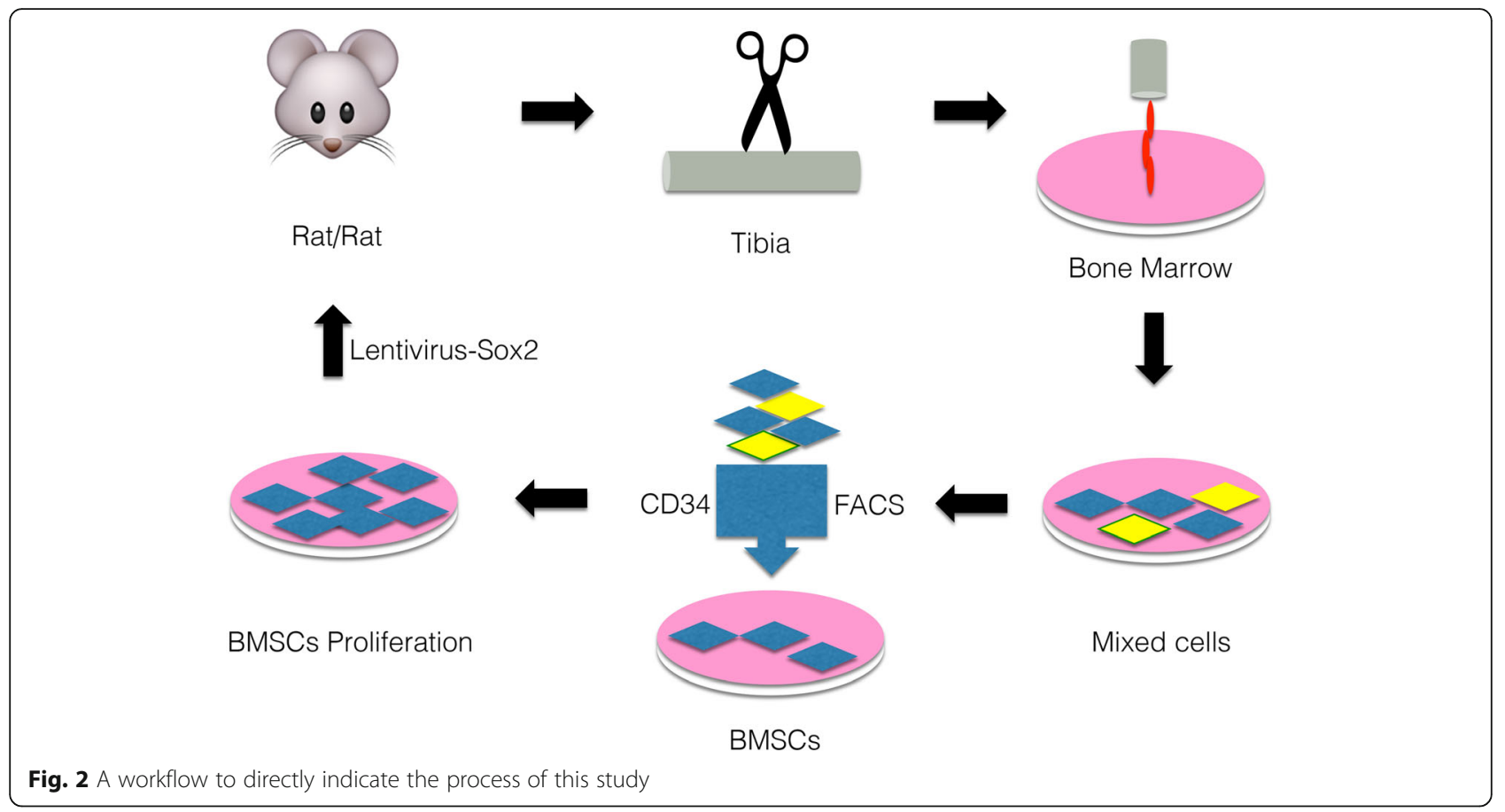

general culture medium were negative for both Oil red $\mathrm{O}$ and Alizarin red S staining (Fig. 3). Therefore, the BMSCs used in our study possessed the general characteristics of bone marrow stem cells.

\section{Ectopic expression of the Sox 2 gene enhanced neuronal differentiation of BMSCs}

After 7 days, the Sox2-BMSCs had large cell body, several short dendrites, and one long tubular axon located in both sides of the cell body, which were similar to common neurons. Immunohistochemistry results showed that Tuj1 was expressed in cells with axon-like processes, which were also GFP positive (Fig. 4). We speculated that the cells with long processes were neuronal-like cells. These results suggested that the ectopic expression of Sox 2 could enhance BMSCs to differentiate into neurons.

\section{Neurological severity score}

The TBI rats were treated with BMSC-Sox2, BMSC, Sox2, and PBS for 7 days later, and the motor function was tested using neurological severity score (NSS). Results showed that NSS were $3.352 \pm 0.398$ in the BMSC-Sox2 group, $4.013 \pm 0.495$ in BMSC group, 4.968 \pm 0.293 in Sox 2 group, and $6.257 \pm 0.361$ in the PBS group. There were significant improvements in the neurological function in the treatment group compared with the PBS group, $p<0.05$. In addition, the
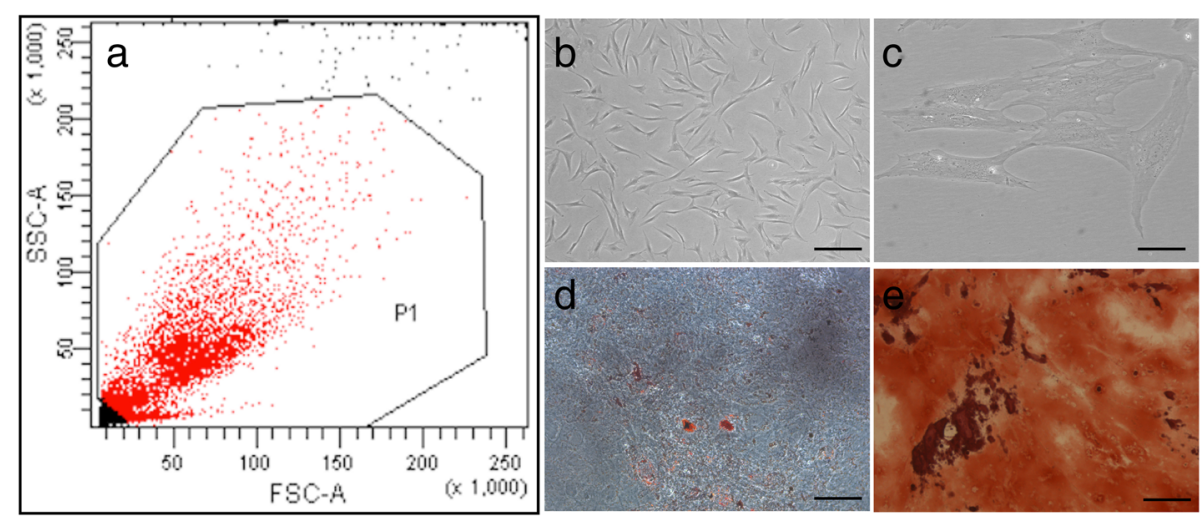

Fig. 3 Identification of characteristics of BMSCs. a Selected by FACS. b, c Morphology of BMSCs. The scale bar = $50 \mathrm{~mm}$. d, e Differentiation of transduced BMSCs. BMSCs were incubated in either adipogenic or osteogenic differentiation medium. The cells were fixed with $4 \%$ paraformaldehyde and stained with Oil Red O for adipogenic cells (d) or Alizarin Red for osteogenic cells (e) 3 weeks in vitro. The scale bar $=50 \mathrm{~mm}$ 


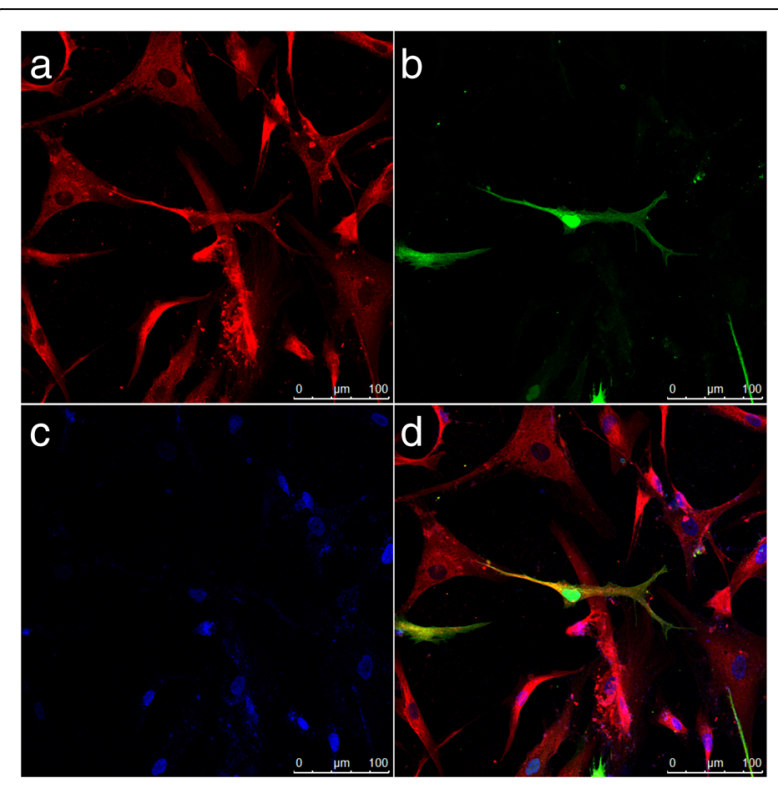

Fig. 4 BMSC-Sox2 transdifferentiated into neurons. a Red is Tuj1 positive. $\mathbf{b}$ Green is rhGFP-Sox2-positive cells. c Blue is nuclei stained by DAPI. d Merge. Scale bar $=100 \mu \mathrm{m}$. Scale bar $=100 \mu \mathrm{m}$

BMSC-Sox2 group had the lowest scores, and there were significant differences between these groups, $p<0.05$ (Fig. 5).

\section{Discussion}

TBI as a significant health concern takes enormous socioeconomic burden for our society. Unfortunately, to date, there are no pharmacologic agents demonstrated to improve TBI outcomes effectively. Therefore, there is a compelling need to develop treatments for TBI. The safety and feasibility of BMSC transplantation are confirmed in animal models and human trials. Our study found that cell therapies using BMSC transplantation combined with over-expression of Sox 2 could improve neurological function in TBI rats, which provided basic support for the treatment of TBI (Fig. 6).

Our study was to evaluate the potential positive benefits of BMSCs with Sox 2 therapy in a rat TBI model. Results showed that BMSCs with Sox 2 promoted the recovery of motor function in a rat TBI model and got better outcomes. In addition, treatment of BMSCs alone and Sox 2 alone also has accurate recovery of motor function, indicating that the BMSCs may provide cell sources for the re-contribution of neural function.

The adoption of BMSCs in the treatment of TBI has taken great benefits in the past years [1,11-13]. BMSCs are not only easy to harvest but also have no immune response. More importantly, they have the ability of differentiation into cells of neuronal lineages and promote repair of neural tissues after TBI. As we have known, Sox2 functions as a core factor for stem cell pluripotency along with Oct4 and Nanog [14]. Sox 2 plays a role in the development of neural progenitors in the CNS. Our data suggest that Sox 2 has a role in the activation of proneural of cell fate determination.

From the above results, we can see that the recovery of nerve function requires a structural basis of functional

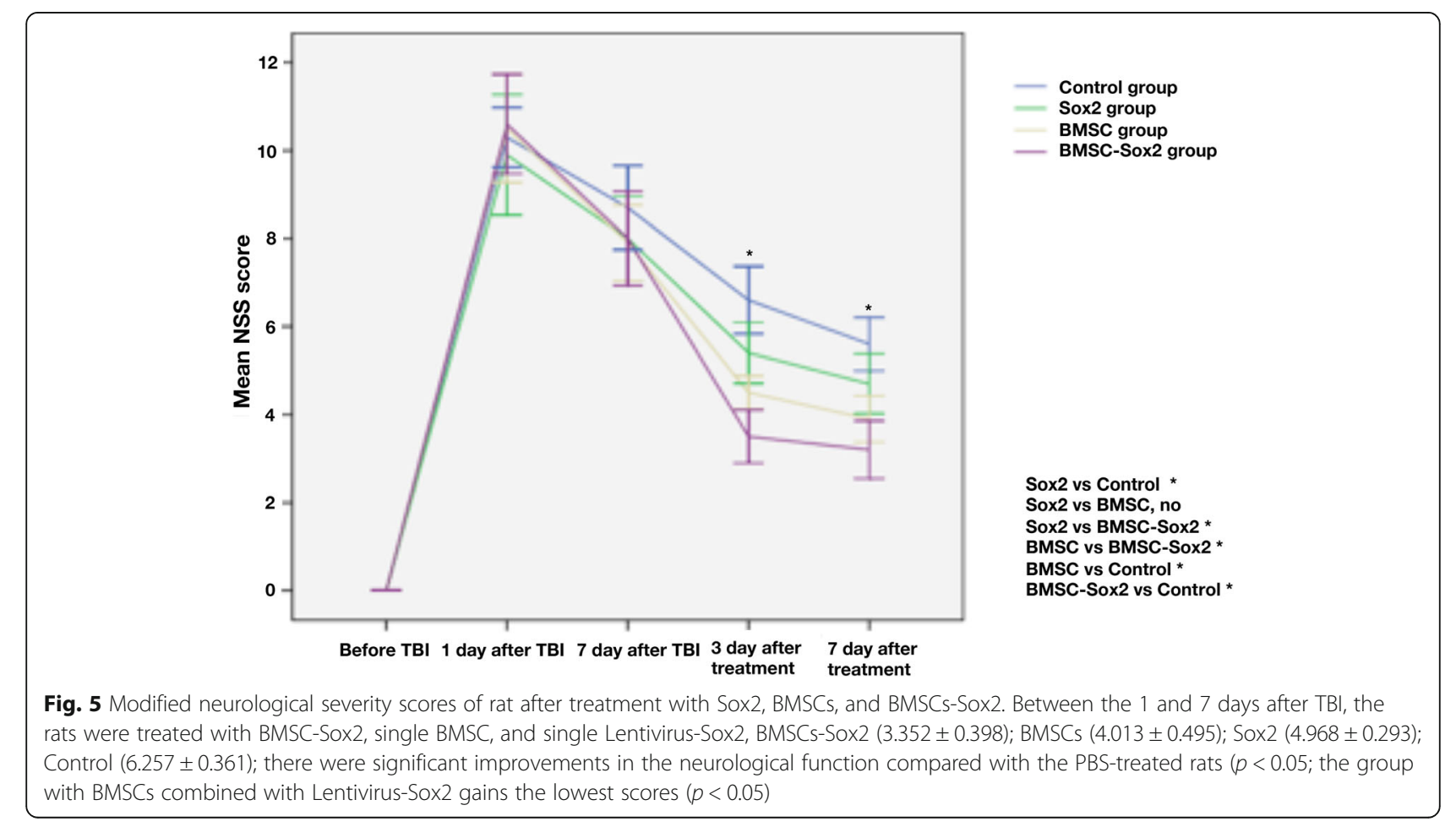




\author{
Raising the rat by the tail 3 \\ 1 Flexion of forelimb \\ 1 Flexion of hindlimb \\ 1 Head moved more than 10 degrees to the \\ vertical axis within $30 \mathrm{~s}$ \\ Walking on the floor 3 \\ 0 Normal walk \\ 1 Inability to walk straight \\ 2 Circling toward the paretic side \\ 3 Fall down to the paretic side \\ Sensory tests 2 \\ 1 Placing test (visual and tactile test) \\ 2 Proprioceptive test (deep sensation, pushing \\ the paw against the table edge to stimulate \\ limb muscles) \\ Beam balance tests 6 \\ 0 Balances with steady posture \\ 1 Grasps side of beam
}

2 Hugs the beam and one limb falls down

from the beam

3 Hugs the beam and two limbs fall down

from the beam or spins on beam $(<60 \mathrm{~s})$

4 Attempts to balance on the beam but falls

off $(<\mathbf{4 0} s)$

5 Attempts to balance on the beam but

falls off $(<20 \mathrm{~s})$

6 Falls off, no attempt to balance of hang onto the beam

Reflexes absent and abnormal movements 4

1 Pinna reflex (head shake when touching

the auditory meatus)

1 Corneal reflex (eye blink when lightly

touching the cornea with cotton)

1 Startle reflex (motor response to a brief

noise from snapping a clipboard and paper)

1 Seizures, myoclonus, myodystony

Maximum no. of points 18

\section{Modified neurological severity score}

Fig 6 Modified neurological severity score for motor function in rats

recovery in TBI, which requires sufficient cell sources to reconstruct the damaged brain tissues. However, it is not efficient to just have a cellular basis, because of the limited ability of differentiation into neurons and other types of BMSCs. Therefore, it also needs ectopic factors to enhance the ability of differentiation into neurons and other types of BMSCs. Based on the above consideration, Sox 2 owns the characteristics promoting the transdifferentiation of BMSCs into neurons and other types of cells. The results also demonstrated that in the BMSC-Sox2 group, rats with TBI have a significant improvement in the recovery of motor function.

In summary, treatment of combining BMSC and Sox2 transplantation has obtained obviously beneficial effects in neural re-contribution in a rat TBI model. However, it remains a need to explore the full effects of BMSC transplantation. More researches are required to explore the other characteristics of BMSCs for clinical applications.

\section{Conclusion}

Sox2 can enhance the ability of differentiation of BMSCs into neurons and other cells and accelerate the recovery of motor function of rats with traumatic brain injury. BMSCs with Sox 2 transplantation can promote the recovery of motor function in a rat TBI model and got better outcomes, providing support for the treatment of TBI in clinic.
Acknowledgements

Thanks to Pro Yan Gao and Pro Duan WM who helped us to review the manuscript and provide constructive advice in the part of the discussion.

\section{Funding}

This study was supported by grant nos. PX2016034 to HW from Beijing Municipal Administration of Hospitals Incubating Program.

\section{Authors' contributions}

$\mathrm{HW}$ designed the whole experiment, and $\mathrm{QH}$ participated in the whole procedure and wrote this manuscript. JZ and YH took part in the BMSC culturing. All authors read and approved the final manuscript.

\section{Ethics approval and consent to participate}

This study was approved by the Animal Use and Care Committee of CMU (AEEl-2018-152). Rats used in the experiment were performed following the National Institutes of Health Guide for the Care and Use of Laboratory Animals.

Consent for publication

Not applicable.

Competing interests

The authors declare that they have no competing interests.

\section{Author details}

${ }^{1}$ Department of Neurosurgery, Beijing Tiantan Hospital, Capital Medical University, No.119 South 4th Ring West Road, Fengtai District, Beijing 100070, China. ${ }^{2}$ Department of Cardiopulmonary Function Examination, Shanxi Provincial Cancer Hospital, Employee Xincun No.3, Xing Hua Ling District, Taiyuan 030000, China. ${ }^{3}$ Basic Medical Science Department, Capital Medical University, You An Men Wai Street, Xi Tou Tiao 10\#, Fengtai District, Beijing 100069, China.

Received: 20 January 2019 Accepted: 9 April 2019

Published online: 15 May 2019

References

1. Hasan A, Deeb G, Rahal R, Atwi K, Mondello S, Marei HE, et al. Mesenchymal stem cells in the treatment of traumatic brain injury. Front Neurol. 2017; 20(8):28. 
2. Hyder AA, Wunderlich CA, Puvanachandra P, Gururaj G, Kobusingye OC. The impact of traumatic brain injuries: a global perspective. NeuroRehabilitation. 2007;22:341-53.

3. Hornbeck K, Walter K, Myrvik M. Should potential risk of chronic traumatic encephalopathy be discussed with young athletes? AMA J Ethics. 2017;19: 686-92.

4. Jiang J-Y, Gao G-Y, Feng J-F, Mao Q, Chen LG, Yang XF, et al. Traumatic brain injury in China. Lancet Neurol. 2019;18:286-95.

5. Yang WH, Yang C, Xue YQ, Lu T, Reiser J, Zhao LR, et al. Regulated expression of lentivirus-mediated GDNF in human bone marrow-derived mesenchymal stem cells and its neuroprotection on dopaminergic cells in vitro. PLoS One. 2013;8:e64389.

6. Hao Q, An JQ, Hao F, Yang C, Lu T, Qu TY, et al. Inducible lentivirusmediated expression of the Oct 4 gene affects multilineage differentiation of adult human bone marrow-derived mesenchymal stem cells. Cell Reprogram. 2015;17:347-59.

7. Narayan S, Bryant G, Shah S, Berrozpe G, Ptashne M. OCT4 and SOX2 work as transcriptional activators in reprogramming human fibroblasts. Cell Rep. 2017;20:1585-96.

8. Zhang X, Cao H, Bai S, Huo W, Ma Y. Differentiation and characterization of rhesus monkey atrial and ventricular cardiomyocytes from induced pluripotent stem cells. Stem Cell Res. 2017:20:21-9.

9. Kalish BT, Whalen MJ. Weight drop models in traumatic brain injury. Methods Mol Biol. 2016;1462:193-209.

10. Zhao S, Yu Z, Liu Y, Bai Y, Jiang Y, Leyen V, et al. CD47 deficiency improves neurological outcomes of traumatic brain injury in mice. Neurosci Lett. 2017;643:125-30

11. Luo XH, Chang YJ, Huang XJ. Improving cytomegalovirus-specific T cell reconstitution after haploidentical stem cell transplantation. J Immunol Res. 2014;14:631951.

12. Guo $S$, Zhen $Y$, Wang A. Transplantation of bone mesenchymal stem cells promotes angiogenesis and improves neurological function after traumatic brain injury in mouse. Neuropsychiatr Dis Treat. 2017;13:2757-65.

13. Wei L, Wei ZZ, Jiang MQ, Mohamad O, Yu SP. Stem cell transplantation therapy for multifaceted therapeutic benefits after stroke. Prog Neurobiol. 2017;157:49-78.

14. Chew IL, Loh YH, Zhang W, Chen X, Tam WL, Yeap LS, et al. Reciprocal transcriptional regulation of Pou5f1 and Sox2 via the Oct4/Sox2 complex in embryonic stem cells. Mol Cell Biol. 2005;25:6031-46.

Ready to submit your research? Choose BMC and benefit from:

- fast, convenient online submission

- thorough peer review by experienced researchers in your field

- rapid publication on acceptance

- support for research data, including large and complex data types

- gold Open Access which fosters wider collaboration and increased citations

- maximum visibility for your research: over $100 \mathrm{M}$ website views per year

At BMC, research is always in progress.

Learn more biomedcentral.com/submissions 